2. Медведкова, И.Е. Базы данных / И.Е. Медведкова, Ю.В. Бугаев, С.В. Чикунов ; Министерство образования и науки РФ, ФГБОУ ВПО «Воронежский государственный университет инженерных технологий» ; науч. ред. Г.В. Абрамов. - Воронеж : Воронежский государственный университет инженерных технологий, 2014. - 105 с. : ил. - Библиогр. в кн. - ISBN 978-5-00032-060-0 ;

3. Узнайте все о Firebird за 2 минуты [Электронный ресурс] // Carlos H. Cantu версия 1.3, февраль 2010 года URL: http://www.firebirdnews.org/docs/fb2min_ru.html (дата обращения: 15.01.2017).

4. Embarcadero RAD Studio 2010 [Электронный ресурс] // Программирование Паскаль (Pascal) и Делфи (Delphi) // URL: http://delphi-box.ru/embarcadero-rad-studio2010.html (дата обращения: 20.02.2017).

\title{
Сравнительный анализ операционных систем iOS и Android
}

\author{
Покииванов Д.О., студент, \\ Технический институт (филиал) \\ Северо-Восточного федерального университета, \\ 2. Нерюнгри \\ E-mail: dmposkhiwanov@gmail.com
}

Научный руководитель: стариий преподаватель Соболева Н.И.

В мире электронных устройств существует большое количество операционных систем для мобильных устройств: iOS, Android, Windows Phone и другие, в настоящее время самыми популярными являются iOS и Andoroid. Именно эти операционные системы удерживают более половины рынка мобильных телефонов, поэтому сравнительный анализ iOS и Android является необходимым. Практически у каждого человека, покупающего мобильное устройство, может возникнуть вопрос о выборе популярной операционной системы.

Общие сведения. IOS - операционная система мобильных телефонов и электронных планшетов, разработанная компанией Apple и появившаяся на свет в 2007 году; изначально - для iPhone и iPod touch, чуть позже — для iPad, Apple TV, iWatch. В отличие от Windows Phone и Android, выпускается только для устройств, производимых компанией Apple.

iOS основывается на ядре XNU, работающее на микроядре Mach и включающее программный код, созданный фирмой Apple, а также код из OC NeXTSTEP и FreeBSD. Ядро iOS практически идентично ядру операционной системы Apple macOS (изначально называвшейся OS X). Начиная с самой первой версии, iOS работает только на планшетных компьютерах и смартфонах с процессорами архитектуры ARM.

Пользовательский интерфейс $\mathrm{IOS}$ основан на концепции непосредственного взаимодействия с применением жестов «мультитач». Компоненты интерфейса включают в себя ползунки, переключатели и кнопки.

iOS создана на основе операционной системы OS X (переименованной позже в $\underline{\operatorname{macOS}})$ и использует тот же набор компонентов Darwin, совместимый со стандартом POSIX. 
Операционная система iPhone OS презентована 9 января 2007 года вместе с смарфоном iPhone собственнолично Стивом Джобсом на конференции Мacworld Conference \& Ехро и выпущена в июне того же года. Компания не подразумевала отдельного названия для операционной системы, поэтому первоначальный слоган звучал таким образом: «іPhone работает на OS X».

По состоянию на 1 мая 2016 года магазин мобильных приложений App Store содержит более 3 млн приложений для iOS, которые были загружены более 250 миллиардов раз.

Прочие приложения могут быть разработаны с помощью Хсоde для Мас и iPhone, iPod Touch и iPad, Codea для iPad, и выгружены в App Store - онлайн-магазин, который внедрен в сам iPhone/iPod touch/iPad, начиная с версии iPhone OS 2.0, и считается крупнейшим магазином мобильных приложений (на июнь 2016 года - более 1 млн. 800 тыс. приложений для iOS, более 600 тыс. приложений специально для iPad).[1]

Android - операционная система смартфонов, интернет-планшетов, электронных книг, наручных часов, игровых приставок и других устройств. В перспективе планируется поддержка автомобилями и бытовыми роботами. Разработана на ядре Linux и реализации виртуальной машины Java от Google. Первоначально разрабатывалась фирмой Android, Inc., которую потом приобрела Google. Позже Google стимулировала создание альянса Open Handset Alliance (OHA), который в настоящее время занимается поддержкой и дальнейшим развитием платформы. Android дает возможность создавать Јаvа-приложения через разработанные компанией Google библиотеки. Android Native Development Kit позволяет внедрять библиотеки и компоненты приложений, написанные на Си и других языках.

В 81.5\% мобильных телефонов, проданных во втором квартале 2016 года, была установлена операционная система Android. При этом за весь 2015 год было реализовано более 1.5 миллиарда устройств на Android.

Google дает возможность свободного скачивания инструментария для разработки (Software Development Kit), который предназначен для х86-машин под операционными системами Linux, Mac OS X (10.4.8 или выше), Windows XP (и старше). Для разработки требуется JDK 5 или новее.[2]

Интерфейс. В настоящее время обе операционные системы пришли к модному плоскому стилю оформления. В каждой из них имеются свои особенности, так в Android включается «материальный дизайн», который сформирован на иконографике. Увидев интерфейс iOS или Android, пользователь сразу поймет, какая перед ним система, несмотря на то, что интерфейс у обоих плоский.

iPhone и iPad не имеют рабочего стола как такового. Вместо него несколько страниц с иконками всех установленных приложений, которые можно вручную сортировать по папкам. На нижней панели располагаются от трех до пяти иконок. Слайд в центре экрана сверху вниз открывает окно Spotlight - поиск контента по устройству, который ищет не только по названию, но в сообщениях, заметках и так далее.

Слайд с верхнего края экрана вниз открывает дополнительное меню, где с помощью виджетов можно настроить доступ к самым важным приложениям или отдельным их функциям, просматривать полезную информацию (погода, курсы и т.д.). В соседней вкладке расположились уведомления. С нижнего края можно «вытянуть» 
меню с тумблерами беспроводных сетей, поворота экрана, с кнопками управления плеера и запуском стандартных приложений: калькулятор, фонарик и т.п.

На планшетах и смартфонах Android есть несколько рабочих столов, количество которых устанавливает пользователь и меню с иконками всех приложений. На рабочих экранах можно устанавливать ярлыки и виджеты как встроенные, так и от сторонних разработчиков. Любой стол можно настроить под свои нужды, расположив там все, что нужно. Верхнее меню с уведомлениями выглядит иначе, чем на iOS, но выполняет те же функции. Там же располагаются и тумблеры.

Приложения и магазин приложений. Ранее преимуществом iOS было количество приложений, доступных для скачивания в виртуальном магазине. Но на текущий момент Google Play догнал конкурента. Оба магазина предлагают своим пользователям 1.5 миллиона приложений. Но любого владельца планшета или смартфона интересует в первую очередь качество программного обеспечения. В этом вопросе борьбы iOS против Android безоговорочной победы добивается компания Apple. B App Store крайне трудно найти приложение, которое не работало бы вообще или «радовало» бы пользователей слабой оптимизацией и большим количеством ошибок. Строгие требования Apple заставляют разработчиков публиковать только качественные продукты, но и сам процесс создания программ максимально упрощен удобным devkit’oм и соответствующей документацией. [3]

Android в свою очередь отвечает более широким выбором бесплатных приложений, а также низкими ценами. Правда, в таком подходе есть и минус - громкие проекты выходят сначала на iOS, а уже потом на платформе от Google.

Сами же магазины приложений достаточно удобны, обладают удобным меню категорий, а страницы самих программ насыщены информацией, скриншотами и видео. Небольшое преимущество тут снова у iOS, так как в App Store структура более эргономична, и интуитивно ее понять гораздо проще. Плюс к этому - раздел рекомендаций, который часто советует скачать довольно интересные конкретному пользователю продукты.

Связь. IOS постепенно расширяет свой функционал за счет дополнительных возможностей iMessage и FaceTime. Первое приложение включает в себя центр отправки стандартных SMS и дополнительной бесплатный сервис обмена сообщениями, который доступен для всех владельцев устройств на базе iOS или Mac. Кроме текстовых сообщений в iMessage можно отправлять свои координаты, фотографии, видео и звуковые сообщения. FaceTime в свою очередь является таким же бесплатным приложением, но для аудио-видеозвонков использует интернетсоединение.

Android не долго отставал и получил от Google обновление телефона и SMSприложения. Сервис Hangouts был встроен в операционную систему, подарив владельцам планшетов и смартфонов тот функционал, который есть у поклонников Apple. Особенностью этого приложения является возможность использования его на ПК, Мас и іOS.

Передача данных. Устройства на iOS или Android всегда обладают модулями WiFi и Bluetooth, но функционал у них разный. Устройства Apple используют Bluetooth лишь для подключения аксессуаров, а пользователи Android могут передавать файлы и другие данные. Еще одним плюсом Android будет использование технологии NFC, которая позволяет передать файл или информацию, провести оплату или поделиться ссылкой, поднеся одно устройство к другому. Apple в ответ разработала собственную 
платежную систему Apple Pay, но ее распространение будет очень медленным. Этот сервис подходит только для платежей, что не делает конкуренцию NFC.

Цены и доступность. Планшеты и смартфоны на базе операционной системы Android выпускают практически все компании, которые, так или иначе, связаны с рынком портативных устройств. За счет этого рынок Google-устройств разбит на множество классов и подклассов, что позволяет покупателям выбирать устройства на свой вкус и уровень дохода. Apple же выпускает только 4 устройства в год - два смартфона, отличающиеся размером диагонали дисплея, и два планшета, один из которых является младшей версией основного как по техническим характеристикам, так и по размеру экрана. При этом после релиза новинок можно еще какое-то время официально приобрести представителей предыдущего поколения, что превращает аргумент об отсутствии ценового выбора в продукции Apple в миф. Кроме того, часто компания продает смартфон еще более ранней версии с урезанной памятью, но это касается только рынка развивающихся стран.

Настройки и персонализация. С каждым годом обе ОС становятся все ближе друг к другу. Ярким доказательством этого являются меню настроек. На аппаратах Apple этот раздел операционной системы выглядит практически идеально: здесь невозможно запутаться, все находится там, где должно быть, а визуальное оформление только подчеркивает это, упрощая навигацию и поиск.

B Android все не так ясно и понятно, но различных разделов, переключателей и полосок регулировки гораздо больше. И это касается стандартных настроек операционной системы. Почти у всех производителей есть свой взгляд на это меню, поэтому часто оно обрастает дополнительными возможностями, превращаясь в полноценный конструктор, позволяющий настроить работу аппарата так, как хочется его владельцу.

Безопасность. Из-за получения root-доступа вытекает множество проблем. iOS и Android - это схватка закрытой и открытой операционной системы. Логично предположить, что чрезмерная открытость ведет к повышению шанса вирусных атак, кражи данных и пр.

Кроме стандартного магазина приложений на Android есть и сторонние. Часть из них предлагается производителями, часть разрабатывается независимыми компаниями. Пользователей это радует расширением возможностей по выбору приложений, а на деле это только ухудшает ситуацию.

B iOS же система закрыта, полный доступ получить нельзя, а App Store является единственным источником игровых и программных новинок на устройстве. Исключением является jailbreak (взлом) и магазин суdia, но тут хакерские атаки на свое же устройство - это уж точно вина самого пользователя.

Кроме того, что Android хоть и часто обновляется, тотальное большинство получается апдейты с задержкой или не получают их вообще. Обилие устройств не дает возможности производителям моментально выпускать новые прошивки для всех своих продуктов. У Apple таких проблем нет.

Сравнительный анализ iOS и Android показал, что обе операционные системы являются весьма функциональными. Android дает широчайшие возможности для настройки аппарата «под себя», может использоваться как на бюджетных устройствах, так и на топовых планшетах или смартфонах, обладает высокими показателями эргономичности, но имеет проблемы с безопасностью и рынком программного обеспечения. Если пользователь готов потратить больше денег ради безопасности 
своих личных данных, то лучше сделать выбор в пользу iOS. Простота и безопасность эксплуатации устройств от Apple дополнены потрясающими приложениями, за которые просят не такие большие суммы. У каждого пользователя есть свои потребности, удовлетворить которые в одном случае сможет Android, а в другом — iOS.

\title{
Список литературы:
}

1. Дэйв Марк и др. iOS 6 SDK. Разработка приложений для iPhone, iPad и iPod touch $=$ Beginning iOS 6 Development Exploring the iOS SDK. — М.: «Вильямс», 2013. — 672 c. - ISBN 978-5-8459-1852-9.

2. Коматинэни C., Маклин Д., Хэшими C. Google Android: программирование для мобильных устройств = Pro Android 2. — 1-е изд. - СПб.: Питер, 2011. — 736 с. — ISBN 978-5-459-00530-1.

3. Блог про iPad и другие планшетные компьютеры [Электронный ресурс]. URL: http://rupadblog.com (дата обращения: 05.12.16)

\section{Разработка информационной системы для аэропорта}

\author{
Покииванов Д.О., студент, \\ Технический институт (филиал) \\ Северо-Восточного федерального университета, \\ 2. Нерюнгри \\ E-mail: dmpokshiwanov@gmail.com
}

Научный руководитель: к.М.н., доцент Похорукова М.Ю.

В настоящее время практически в любой организации возникает необходимость организации более эффективной работы, не исключением является и аэропорт. Сегодня воздушный транспорт является наиболее быстрым и удобным видом транспорта при перемещении на далекие расстояния. В мире существуют тысячи аэропортов и соответственно ещё больше маршрутов полетов, поэтому поддержка электронной базы данных со всей необходимой информацией позволит хранить, структурировать информацию и извлекать ее наиболее оптимальным образом.

Перечислим функции, которые должна выполнять разрабатываемая программа:

- просмотр всей информации, которая находится в базе;

• поиск необходимых данных;

- дополнение, удаление, редактирование записей базы данных.

База данных разрабатываемой системы должна удовлетворять информационные потребности и содержать в себе информацию о рейсах, совершаемых авиакомпаниями, вести учет пилотов и осуществлять выдачу подробной информации по запрашиваемому рейсу, обеспечивать расписание вылетов и прилетов воздушных суден, содержать таблицы диспетчеров и выводить график их дежурства.

Для удобного поиска интересующей информации необходимо реализовать:

- сортировку записей;

• фильтрацию записей согласно с введенными значениями отдельных полей или их диапазонов; 